\title{
Synthesis of Chalcopyrite Nanoparticles via Thermal Decomposition of Metal-Thiolate
}

\author{
Takamitsu Kino $^{1, * 1, * 2}$, Toshihiro Kuzuya ${ }^{2}$, Keiichi Itoh ${ }^{1, * 1}$, Kenji Sumiyama ${ }^{1}$, \\ Takahide Wakamatsu² and Minoru Ichidate ${ }^{2}$ \\ ${ }^{1}$ Department of Materials Science and Engineering, Nagoya Institute of Technology, Nagoya 466-8555, Japan \\ ${ }^{2}$ Faculty of Urban Science, Meijo University, Kani 509-0261, Japan
}

\begin{abstract}
$\mathrm{CuInS}_{2}$ nanoparticles (NPs) have been synthesized via thermal decomposition of metal-thiolate in high boiling temperature solvent. This method does not require toxic, unstable and expensive raw materials. Nearly monodispersed NPs with average diameter ranging from 1.8 to $2.8 \mathrm{~nm}$ are obtained. The NPs are found to be compositionally copper rich, $\mathrm{Cu}:$ In $: \mathrm{S}=1.6: 1.0: 1.3$. They exhibit size-dependent optical properties such as photoluminescence (PL) and optical absorption, indicating quantum confinement effects. In PL spectra, the stokes-shifts ( $\sim 300 \mathrm{meV}$ ) from the absorption band edge and their broad PL spectra indicate that PL is ascribed to a donor-acceptor transition. These results demonstrate that chalcopyrite NPs are promising candidates for luminous and solar cell materials. [doi:10.2320/matertrans.MBW200724]
\end{abstract}

(Received October 17, 2007; Accepted November 26, 2007; Published January 30, 2008)

Keywords: chalcopyrite, copper indium sulfide, nanoparticle, dodecanethiol, photoluminescence

\section{Introduction}

Nano-sized materials reveal anomalous optical and catalytic properties for various applications. Semiconductor nano-particles (NPs) are model systems of zero-dimensional quantum confinement and in the last decade novel methods have been proposed to synthesize monodispersed II-VI semiconductor NPs with high quantum yield. ${ }^{1,2)}$ Indeed, IIVI semiconductors such as $\mathrm{CdS}$, CdSe and CdTe are useful for fluorescent probes of bio-medical makers. ${ }^{3)}$ They are also expected to be tunable absorbers and emitters in nanoscale electronics, quantum dot lasers, optical devices and new type solar cells. ${ }^{4)}$ However, the use of toxic heavy metals such as $\mathrm{Cd}, \mathrm{Hg}$ and $\mathrm{Pb}$ must be inhibited.

In this context, ternary chalcopyrites, $\operatorname{CuMX}_{2}(\mathrm{M}=\mathrm{Al}$, $\mathrm{Ga}, \mathrm{In} ; \mathrm{X}=\mathrm{S}, \mathrm{Se}$ ) are alternative materials of II-VI semiconductors, because they are direct-band gap semiconductors. Their optimum band gaps $\left(\mathrm{CuInS}_{2}: \sim 1.5 \mathrm{eV}\right.$, $\left.\mathrm{CuInSe}_{2}: \sim 1.1 \mathrm{eV}\right)$, large absorption coefficients $\left(10^{5} \mathrm{~cm}^{-1}\right)$ and large tolerance for cosmic rays are prominent for absorber layers of hetero-junction solar cells. ${ }^{5-7)}$ Actually a $\mathrm{CuInS}_{2}$ thin-film cell has a quantum efficiency of $12.5 \%{ }^{8)}$ and tunable PL is oberved in $\mathrm{ZnS}-\mathrm{CuInS}_{2}$ mixed crystal NPs. ${ }^{9)}$ Moreover, it has been reported that a $\mathrm{CuInS}_{2}$ quantum dot solar cell has an overall energy conversion efficiency $4 \%{ }^{10)}$ These results encourage us to fabricate chalcopyrite NPs.

In solution phase synthesis, chalcopyrite NPs have been prepared by a TOP/aliphatic amine-S system ${ }^{9)}$ or a thermolysis of single-source precursor such as copper-indium thiolate, ${ }^{11)}\left(\mathrm{PPh}_{3}\right)_{2} \mathrm{AgIn}(\mathrm{SeC}\{\mathrm{O}\} \mathrm{Ph})_{4},{ }^{12)} \quad(\mathrm{TOP})_{2} \mathrm{CuIn}(\mathrm{S}-\mathrm{n}$ $\mathrm{Pr})_{4}{ }^{13)}$ and $\left(\mathrm{PPh}_{3}\right)_{2} \mathrm{CuIn}(\mathrm{SEt})_{4} \cdot{ }^{14)}$ In particular, chalcopyrite NPs with the quantum yield of about $5 \%$ were successfully synthesized via the thermal decomposition of single source precursors. ${ }^{13,14)}$ However, this method has several disadvantages: it is sensitive to atmospheric conditions, needs expensive starting reagents and the methodology becomes

\footnotetext{
${ }^{* 1}$ Graduate Student, Nagoya Institute of Technology

${ }^{* 2}$ Corresponding author, E-mail: kino@ss.emat.nitech.ac.jp
}

cumbersome. In this paper, we present a moderate and facile synthesis route of $\mathrm{CuInS}_{2}$ NPs via thermal decomposition of $\mathrm{Cu}$-In-thiolate at $473 \mathrm{~K}$ without using toxic raw materials. ${ }^{15}$ ) $\mathrm{UV}$-vis and photoluminescence (PL) studies on nearly monodispersed NPs with average diameter ranging from 1.8 to $2.8 \mathrm{~nm}$ evidently indicate quantum confinement effects.

\section{Experimental Procedures}

The following is a typical experimental procedure for synthesis of $\mathrm{CuInS}_{2}$ NPs. All reagents were used as received. $0.4 \mathrm{mmol}$ of copper (II) acetate $\left(\mathrm{Cu}(\text { ace })_{2}\right)$ and $0.4 \mathrm{mmol}$ indium (III) acetate $\left(\operatorname{In}(\text { ace })_{3}\right)$ were mixed with $2.46 \mathrm{ml}$ of 1 dodecanethiol (DT) in tri-n-octylamine (TOCA). After being degassed by Ar gas flushing for $7.2 \mathrm{ks}$, the mixture solution was heated up to $503 \mathrm{~K}$. Then, it was cooled down to room temperature and $30 \mathrm{ml}$ of ethanol was mixed. The precipitates were separated by centrifuging the colloidal solution to remove excess reaction agents and then redispersed in hexane. This precipitate - redispersion procedure was repeated several times to purify the precipitates. For transmission electron microscope (TEM) observation, a drop of $\mathrm{CuInS}_{2}$ colloidal solution was placed on a carbon-coated TEM grid and examined with Hitachi HF-2000 field emission TEM operating at $200 \mathrm{kV}$. The crystal structures of $\mathrm{CuInS}_{2}$ NPs were identified by X-ray diffraction (XRD, Mac Science, $\mathrm{M} 18 \mathrm{XCE}$ ) on sol samples dried onto a glass plates. Chemical composition of prepared NPs were estimated by an EDX analyzer installed in a scanning electron microscope (Hitachi, S-4700 FE-SEM). UV-vis absorption spectra were observed between 200 and $2200 \mathrm{~nm}$ using JASCO V-570 equipped with a PbS detector and PL spectra were recorded on Otsuka Electronics PTI5100S PL-spectrometer at ambient temperature.

\section{Results}

Initial mixture solution turned to be transparent-yellowish 


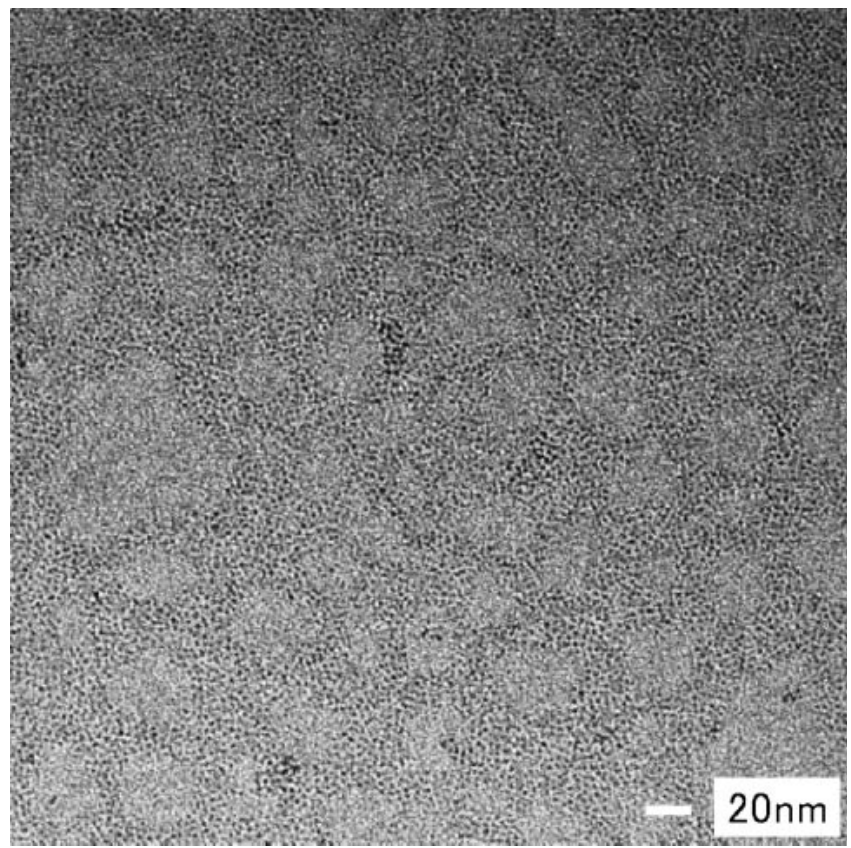

Fig. 1 TEM image of $\mathrm{CuInS}_{2}$ NPs synthesized at $503 \mathrm{~K}$.

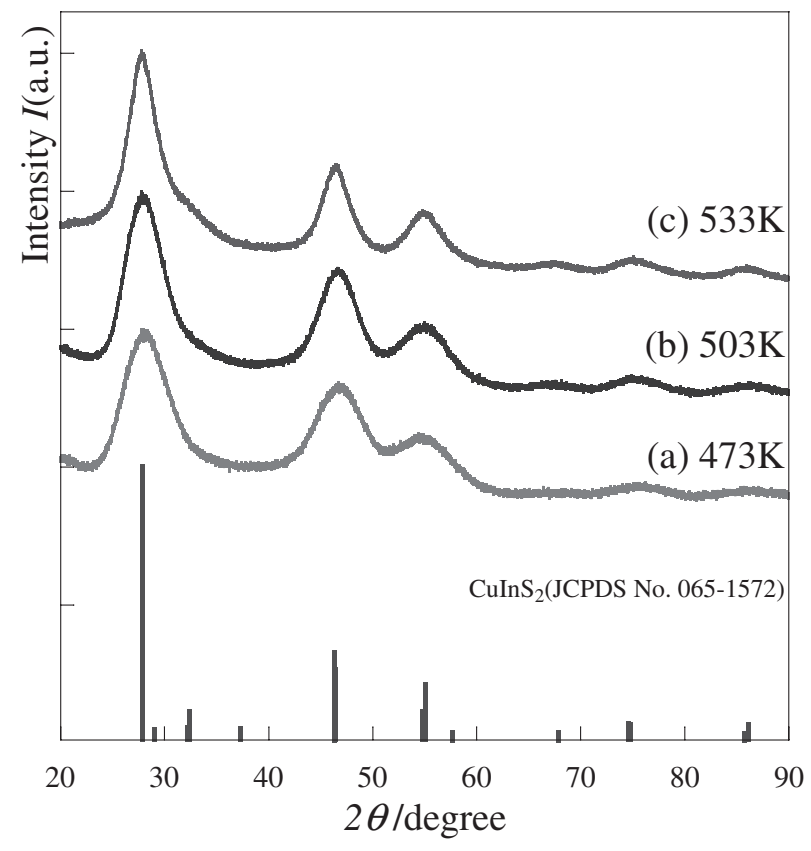

Fig. 2 XRD patterns of $\mathrm{CuInS}_{2}$ NPs synthesized at (a) $473 \mathrm{~K}$, (b) $503 \mathrm{~K}$ and (c) $533 \mathrm{~K}$.

solution at about $353 \mathrm{~K}$. It immediately changed to orange and finally dark red above $473 \mathrm{~K}$. NPs were easily precipitated by polar solvent (ethanol) and redispersed in non-polar solvent such as hexane, benzene and toluene, indicating an NP-surfactant (DT) core-shell structure. TEM image of NPs prepared at $503 \mathrm{~K}$ is shown in Fig. 1. The formation of very fine NPs can be detected.

Figure 2 shows XRD patterns of the NPs obtained at various reaction temperatures, (a) 473, (b) 503 and (c) $533 \mathrm{~K}$. The thermal decomposition of $\mathrm{Cu}$-In-thiolate leads to the

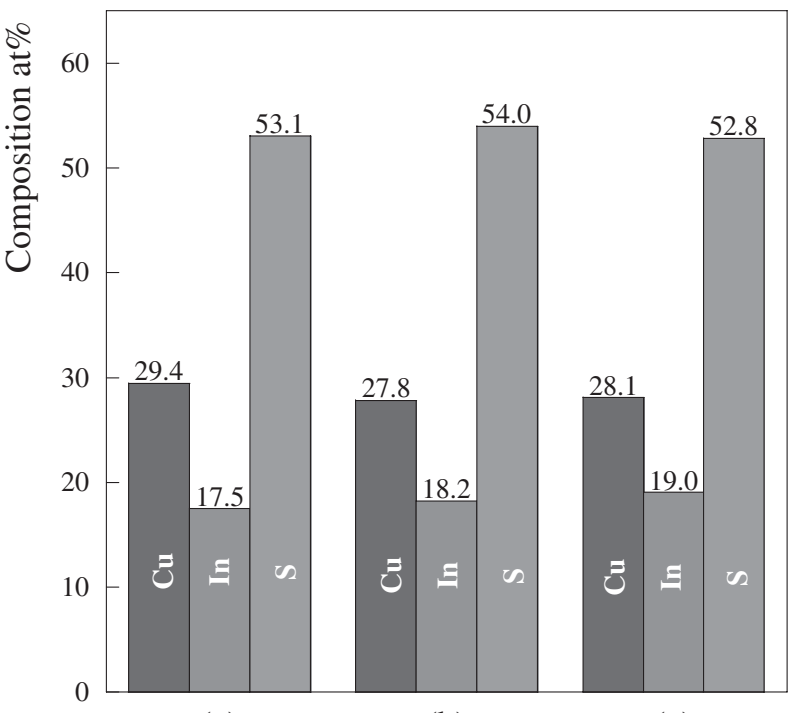

(a)

(b)

(c)

$\mathrm{CuInS}_{2}$ NPs

Fig. 3 Chemical composition of $\mathrm{CuInS}_{2}$ NPs synthesized at (a) $473 \mathrm{~K}$, (b) $503 \mathrm{~K}$ and (c) $533 \mathrm{~K}$.

formation of $\mathrm{CuInS}_{2} \mathrm{NPs}$ with the chalcopyrite structure. The average sizes of NPs estimated by the Scherrer equation are (a) 1.8 , (b) 2.0 and (c) $2.8 \mathrm{~nm}$, being consistent with the TEM image in Fig. 1.

Since optical properties of semiconductor compounds usually depend on the chemical composition, the chemical compositions of synthesized NPs were determined to be $\mathrm{Cu}: \mathrm{In}: \mathrm{S}=$ (a) $1.68: 1: 3.03$, (b) $1.52: 1: 2.96$ and (c) $1.47: 1: 2.74$ (see Fig. 3). For surfactant-capped $\mathrm{CuInS}_{2}$ NPs, Cu-rich layers are presumably deposited on NPs surfaces because of the affinity of capping agent for $\mathrm{Cu}$ atom. ${ }^{13)}$ Therefore, the $\mathrm{Cu}$ to $\mathrm{In}$ ratio increases with the increase in the surface to volume ratio, that is, the decrease in NP's size. This is consistent with our experimental results. Comparing with aliphatic phosphine, ${ }^{13)}$ DT is a suitable capping agent for $\mathrm{CuInS}_{2}$ NPs because the $\mathrm{Cu}$ to In ratio of $\mathrm{CuInS}_{2}$ NPs derived from $\mathrm{Cu}$-In thiolate is closer to the stoichiometry.

Figure 4 shows UV-vis and PL spectra of $\mathrm{CuInS}_{2} \mathrm{NCs}$ obtained at various preparation temperatures, (a) 473, (b) 503 and (c) $533 \mathrm{~K}$. In Fig. 4(a), the UV-vis spectra of $\mathrm{CuInS}_{2} \mathrm{NCs}$ exhibit absorption shoulders at around (a) 2.45, (b) 2.25 and (c) $2.04 \mathrm{eV}$, which were determined from the extremums of the second derivative of absorption spectra. They are attributed to the HOMO-LUMO transition beyond the band gap, where the long absorption tail is also obsrved at the lower energy side.

In Fig. 4 the PL spectra of $\mathrm{CuInS}_{2}$ NCs obtained at 473 , 503 and $533 \mathrm{~K}$ reveal the maxima at (a) 2.00, (b) 1.88 and (c) $1.70 \mathrm{eV}$. The stokes-shifts in the PL spectrum from the band gap ((a) 2.45, (b) 2.25 and (c) $2.04 \mathrm{eV}$ ) are about $0.3 \mathrm{eV}$. Such Stokes shift and its wide full width at half maximums (FWHM) of PL resemble the reported results, ${ }^{13,14)}$ indicating that ambient temperature PL is not attributed to the exciton recombination. 


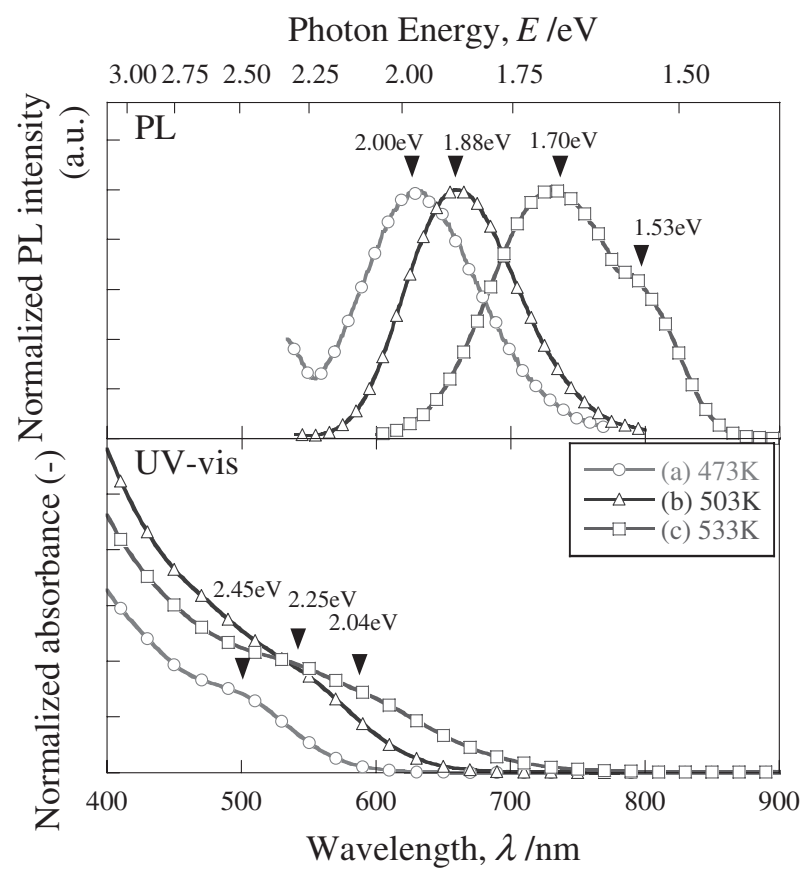

Fig. 4 UV-vis, and PL spectra of $\mathrm{CuInS}_{2}$ NPs synthesized at various reaction temperature. Inset values represent transition energies for UV-vis and PL peak position for PL.

\section{Discussion}

\subsection{Formation processed of $\mathrm{CuInS}_{2} \mathrm{NPs}$}

Our previous results indicated that $\mathrm{Cu}$-thiolate was decomposed into copper sulfide and organic byproducts. ${ }^{16)}$ Since the decomposition of In-thiolate requires higher reaction temperature, possible reaction paths for $\mathrm{CuInS}_{2}$ NPs formation are summarized as follows (Fig. 5).

I: Metal ions, $\mathrm{Cu}^{2+}$ and $\mathrm{In}^{3+}$, react with DT to form bridged complexes (-Cu-SR-In-SR-) $)_{n}$, where $\mathrm{Cu}^{2+}$ is reduced to a monovalent ion. Then, thermal decomposition of (-Cu-SRIn-SR- $)_{n}$ leads to formation of $\mathrm{CuInS}_{2}$ monomers and their polymerization (crystal growth).

II: $\mathrm{Cu}-\mathrm{SR}$ complexes are decomposed into $\mathrm{Cu}_{2} \mathrm{~S}$ monomers. Then, $\mathrm{Cu}_{2} \mathrm{~S}$ monomers react with In-SR complexes to form $\mathrm{CuInS}_{2}$ monomers.

These paths, I and II, are influenced by their coordinating solvent and/or capping agent (co-ligand). In highly coordi- nating solvent (such as tri-n-octylphosphine and di-octylamine) systems, relatively large NPs with a wurtzite structure (hexagonal), have been obtained. ${ }^{17)} \mathrm{A}$ masking effect by highly coordinating solvent prevents the formation of $(-\mathrm{Cu}-$ SR-In-SR-) ${ }_{n}$ complexes (in path I) and/or the reaction between $\mathrm{Cu}_{2} \mathrm{~S}$ monomers and In-SR complexes (in path II). In order to clarify the real reaction path, we need further detailed investigation of NPs growth kinetics using mass and in-situ UV-vis spectroscopy.

\subsection{Optical properties of $\mathrm{CuInS}_{2}$ NPs}

UV-vis spectra show typical curves for semiconductor materials, where the band gap energy larger than $1.53 \mathrm{eV}$ has been reported for $\mathrm{CuInS}_{2}$ bulk materials. ${ }^{18)}$ Based on the conventional quantum confinement theory, the following equation has been used to calculate the gap energy of $\mathrm{CuInS}_{2}$ NPs,

$$
\Delta E_{\mathrm{g}}=\frac{h^{2} \pi^{2}}{2 \mu R}-\frac{1.786 e^{2}}{\varepsilon_{1} R}-\frac{0.124 \times 2 \mu}{m_{0} \varepsilon_{1}^{2}} \times 13.6058,
$$

where $R$ is the radius of NPs, $\mu=1 / m_{\mathrm{e}}{ }^{*}+1 / m_{\mathrm{h}}{ }^{*}\left(m_{\mathrm{e}}{ }^{*}\right.$, $m_{\mathrm{h}}{ }^{*}=$ effective masses of electron and hole) and $\varepsilon_{1}$ is the dielectric constant of NP. Adopting $\mu=0.14 m_{0}\left(m_{\mathrm{o}}=\right.$ free electron mass) and $\varepsilon_{1}=8.5, E_{\mathrm{g}, \mathrm{cal}}$ are estimated to be (a) 4.5 , (b) 3.9 and (c) $2.7 \mathrm{eV}$ for $\mathrm{CuInS}_{2}$ NPs. ${ }^{18)}$ These values are inconsistent with the experimental results because the formula does not take into account of surface effects of NPs'. Monodispersed semiconductor NPs usually exhibit a sharp UV-vis absorption feature, while the present $\mathrm{CuInS}_{2}$ NCs have an indistinct absorption peak and a long absorption tail on the lower energy side. Though these results suggest the poor size uniformities of $\mathrm{CuInS}_{2} \mathrm{NCs}$, the broad absorption peak is also observed even in monodispersed $\mathrm{CuInS}_{2} \mathrm{NPs}$ whose standard deviation is $\sim 11 \%$. The excitonic spectra of these monodispersed NPs indicate the presence of several intraband. ${ }^{14,17)}$

The PL effect has been detected in $\mathrm{CuInS}_{2}$ NPs at room temperature and easily confirmed with the naked eye. Moreover, PL spectra exhibit a marked stokes-shift, where the energy difference between the absorption and emission peaks are larger than a binding energy of an exciton pair $(\sim 0.02 \mathrm{eV})$. In $\mathrm{CuInS}_{2}$ crystals which contain various sorts of defects, vacancies, substitutional and interstitial sites, there are intraband states such as donor and acceptor levels. It has

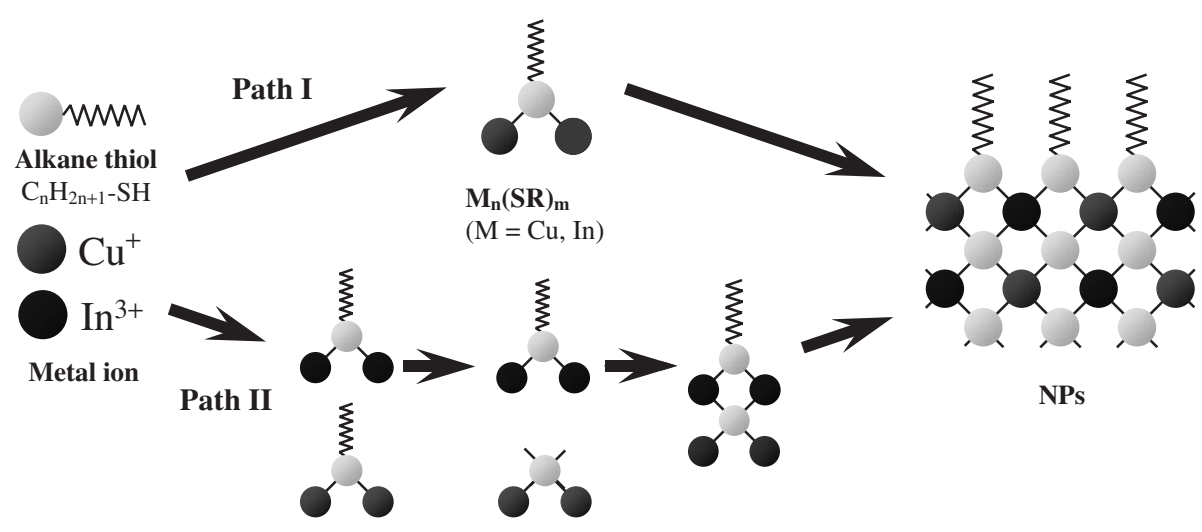

Fig. 5 Reaction scheme for the synthesis process. 
been proposed that $\mathrm{CuInS}_{2}$ crystals have two donor and two acceptor states; ${ }^{14)}$ a sulfur vacancy level (D1), an In interstitial (or Cu-In substitution) level (D2), acceptor levels for $\mathrm{Cu}$-rich (A1) and for In-rich (A2). The transition energies from these donor states to acceptor states are estimated by the following equation: ${ }^{14)}$

$$
h v=E_{\mathrm{g}}-E_{\mathrm{A}}-E_{\mathrm{D}}+e^{2} / 4 \pi \varepsilon r,
$$

where $E_{\mathrm{A}}, E_{\mathrm{D}}(\mathrm{A} 1: 0.15, \mathrm{~A} 2 ; 0.1 \mathrm{eV}, \mathrm{D} 1: 0.035, \mathrm{D} 2: 0.07)$ and $\mathrm{r}$ are the activation energies of acceptor, donor and the distance between the acceptor and donor sites, respectively. Assuming that PL is originated from the donor-acceptor pair recombination, the stokes-shift in the emission wavelength is $0.14 \sim 0.22 \mathrm{eV}$, being smaller than the experimental results of about $0.3 \mathrm{eV}$. Moreover, the PL intensity is very sensitive to surface because the surfactants on $\mathrm{CuInS}_{2}$ NPs eliminate surface-dangling bonds. When capping agents such as DT, fatty acid and aliphatic amine etc are added to the $\mathrm{CuInS}_{2}$ colloidal solution, the PL intensity is enhanced, while it is suppressed by addition of excessive capping agents. Therefore, the large stokes-shift is ascribed to the unique surface structure of $\mathrm{Cu}$-rich layers of NPs, where the donor-acceptor recombination originates from intrinsic defects and surface dangling bonds cause radiationless transitions.

\section{Summary}

We have developed the facile synthesis route of $\mathrm{CuInS}_{2}$ NPs via thermal decomposition in low-coordinating solvent. Very fine $\mathrm{CuInS}_{2}$ NPs with a chalcopyrite structure can be obtained. They exhibit a marked PL effect at room temperature and its wavelength varies within $1.7 \sim 2.0 \mathrm{eV}$. These stokes shifts and broad FWHM suggest that the observed PL is attributed to the donor-acceptor recombination in the $\mathrm{Cu}$ rich surface layer.

\section{Acknowledgement}

The authors wish to express their sincere thanks to Prof. $\mathrm{H}$. Masuda for their use of a PL spectrometer and Dr. Y. Hamanaka for discussion of optical properties. This work was supported by a grant from the NITECH 21 st Centry COE
Program, "World Ceramics Center for Environmental Harmony", Meijo University Open Research Center project, and Intellectual Cluster Project of Aichi-Nagoya Area given by the Ministry of Education, Science, Culture and Sports, Japan.

\section{REFERENCES}

1) C. B. Murray, C. R. Kagan and M. G. Bawendi: Science 270 (1995) 1335-1338.

2) A. P. Alivisatos: Science 271 (1996) 933-937.

3) W. C. W. Chan and S. Nie: Science 281 (1998) 2016-2018.

4) W. U. Huynh, J. J. Dittmer and A. P. Alivisatos: Science 295 (2002) 2425-2427.

5) S. G. Bailey and D. J. Flood: Prog. Photovolt. Res. Appl. 6 (1998) 1-14.

6) H. W. Schock and R. Noufi: Prog. Photovolt. Res. Appl. 8 (2000) 151160.

7) H. W. Schock and K. Bogus: Development of CIS solar cells for space applications. In Proceedings of the Second World Conference on Photovoltaic Energy: J. Schmid, H. A. Ossenbrink, P. Helm, H. Ehmann, E. D. Dunlop, Eds. EC Joint Research Center: Luxembourg, July (1998) 3586-3589.

8) J. Klaer, J. Bruns, R. Henninger, K. Topper, R. Klenk, K. Ellmer, D. Braunig, A tolerant two step process for efficient $\mathrm{CuInS}_{2}$ solar cells. In Proceedings of the Second World conference on Photovoltaic Solar Energy Conversion; J. Schmid, H. A. Ossenbrink, P. Helm, H. Ehmann, E. D. Dunlop, Eds.; EC Joint Research Center: Luxembourg, July (1998) 537-540.

9) H. Nakamura, W. Kato, M. Uehara, K. Nose, T. Omata, S. Otsuka-YaoMatsuo, M. Miyazaki and H. Maeda: Chem. Mater. 18 (2006) 33303335.

10) M. Nanu, J. Schoonman and A. Goossens: Adv. Mater. 16 (2004) 453456.

11) S. Choi, E. Kim and T. Hyeon: J. Am. Chem. Soc. 128 (2006) 25202521.

12) M. T. Ng, C. B. Boothroyd and J. J. Vittal: J. Am. Chem. Soc. 128 (2006) 7118-7119.

13) J. J. Nairn, P. J. Shapiro, B. Twamley, T. Pounds, R. von Wandruszka, T. R. Fletcher, M. Williams, C. Wang and M. G. Norton: Nano Lett. 6 (2006) 1218-1223.

14) S. L. Castro, S. G. Bailey, R. P. Raffaelle, K. K. Banger and A. F. Hepp: Chem. Mater. 15 (2003) 3142-3147.

15) Pattent-JPN-2007-027828, JPN-2007-135374.

16) T. Kuzuya, S. Yamamuro, T. Hihara and K. Sumiyama: Chem. Lett. 33 (2004) 352-353.

17) Manuscript in preparation.

18) A. M. Malyarevich, K. V. Yumashev, N. N. Posnov and V. P. Mikhailov: J. App. Phys. 87 (2000) 212-216. 